\title{
CIRUGÍA DE AGUJERO MACULAR SIN POSICIÓN «BOCA ABAJO» POSTOPERATORIA. ESTUDIO PILOTO
}

\author{
MACULAR HOLE SURGERY WITHOUT POSTOPERATORY \\ «FACE DOWN» POSITIONING. PILOT STUDY
}

\author{
GUERRERO-NARANJO JL ${ }^{1}$, CORTÉS-LUNA C ${ }^{1}$, MORALES-CANTÓN V V', COLINA-LÚQUEZ J ${ }^{1}$, \\ RIVERA-SEMPERTEGUI J ${ }^{1}$, NAVARRO-LÓPEZ $\mathrm{P}^{1}$, DE REGIL $\mathrm{M}^{1}$, BLANCO-MORENO $\mathrm{E}^{1}$, \\ QUIROZ-MERCADO H ${ }^{1}$
}

\begin{abstract}
RESUMEN
Objetivos: Evaluar los resultados visuales y anatómicos en el tratamiento del agujero macular con facoemulsificación, vitrectomía y gas intraocular sin mantenimiento de posición boca abajo en el postoperatorio.

Material y metodos: Se realizó cirugía de facoemulsificación con implante de lente intraocular, vitrectomía con pelado de la membrana limitante interna y gas perfluoropropano $\left(\mathrm{C}_{3} \mathrm{~F}_{8}\right)$ al $17 \%$ en pacientes con diagnóstico de agujero macular grados III y IV. En el postoperatorio se indicó evitar la posición supina durante 15 días. Se realizó intercambio liquido gas en los pacientes con menos del $50 \%$ de gas a la primera semana. Se evaluó la mejoría de la agudeza visual y el porcentaje de cierre del agujero macular después de un año de seguimiento. Resultados: Se incluyeron 20 ojos de 20 pacientes con edad entre 60 y 75 años con una mediana de 68 años. La mejor agudeza visual corregida (AVcc) inicial varió de 0,05 a 0,13 con una mediana de 0,06 . La AVcc final estuvo entre 0,05 a 0,3 con una
\end{abstract}

\begin{abstract}
Purpose: To evaluate the visual and anatomical outcomes of macular hole surgery using phacoemulsification, vitrectomy and intraocular gas tamponade without the use of postoperative face down positioning.

Methods: Phacoemulsification with an intraocular lens implant, followed by vitrectomy with internal limiting membrane peeling and perfluoropropane $\left(\mathrm{C}_{3} \mathrm{~F}_{8}\right)$, was performed in patients with stage 3 or 4 macular holes. After surgery, patients had to avoid the supine position for 15 days. Patients with a gas level $<50 \%$ during the first week had a fluid-gas exchange. Visual acuity and closure of the macular hole were evaluated after 1 year of follow-up.

Results: 20 eyes of 20 patients, aged 60 to 75 years (median age 68 years), were included in this study. The best initial visual acuity ranged from 0.05 to 0.13 (ETDRS), with the median being 0.06 . The final visual acuity was 0.05 to 0.30 with a median of 0.10 . A statistically significant improvement ( $\mathrm{p}=0.001$, Wilcoxon) was found. The anatomic pos-
\end{abstract}

Recibido: 20/12/04. Aceptado: 12/6/06.

Asociación para evitar la ceguera en México. Hospital Luis Sánchez Bulnes. Universidad Nacional Autónoma de México.

1 Doctor en Medicina.

Correspondencia:

José Luis Guerrero Naranjo

Servicio de Retina

Asociación para evitar la ceguera en México

Hospital Luis Sánchez Bulnes

Vicente García Torres No. 46 (Col. San Lucas Coyoacán)

C.P. 04030 México D.F.

México

E-mail: guenarmx@yahoo.com.mx 
mediana de 0,10 . Al comparar la agudeza visual inicial y final se encontró una mejoría estadísticamente significativa de $\mathrm{p}=0.001$ (Wilcoxon). Los resultados postoperatorios en cuanto al estado anatómico final del agujero macular fueron exitosos en el $90 \%$ $(n=18)$ y sin éxito en el $10 \%(n=2)$.

Conclusiones: La cirugía de agujero macular es generalmente beneficiosa y los pacientes pueden mejorar su visión sin ser necesario el mantenimiento de posiciones incomodas e intolerables para algunos pacientes.

Palabras clave: Perforaciones retinianas, vitrectomía, facoemulsificación. toperative results revealed $90 \%(\mathrm{n}=18)$ of the macular holes were closed while $10 \%(\mathrm{n}=2)$ were not.

Conclusions: Macular hole surgery has, in general, good results and patients can achieve improvement in their visual acuity without the need for uncomfortable and unbearable post operative posture positions (Arch Soc Esp Oftalmol 2006; 81: 321-326).

Key words: Retinal perforations, vitrectomy, phacoemulsification.

\section{INTRODUCCIÓN}

El agujero macular afecta a uno de cada 1.000 individuos, de éstos el $72 \%$ son mujeres entre la sexta y séptima década de la vida (1) con un rango de bilateralidad del 6 al 22\%. Los ojos con agujero macular idiopático disminuyen su visión secundariamente a pérdida de tejido, cambios retinianos quísticos, desprendimiento del anillo retiniano que circunda al agujero, y degeneración de los fotorreceptores (2). Gass en 1988 describió la patogénesis del agujero macular idiopático, considerando a la tracción vitreorretiniana tangencial como la causa de esta patología (3). En 1988 Smiddy y colaboradores realizaron esta cirugía sin poder definir la utilidad de la vitrectomía y debido a la progresión de esclerosis del cristalino y complicaciones potenciales en la retina, concluyeron que era una mejor opción el manejo conservador en esta etapa (4). Posteriormente se realizaron estudios en pacientes con agujero macular idiopático grados III o IV en los que la vitrectomía vía pars plana con remoción del vítreo posterior y tamponamiento con gas expansible inducía resolución del agujero macular en un porcentaje del 58 al $97 \%$ de los casos con mejoría visual del 42 al $85 \%(2,5,6)$.

En 1995 Thompson, Glaser et al publicaron que el $76 \%$ de los casos de cirugía de agujero macular requirieron más tarde una extracción de cristalino por progresión de la esclerosis nuclear (7). En 1997 Miller y colaboradores describieron los resultados de 4 casos en los que se usó perfluoropropano al $15 \%$ con cirugía de catarata y colocación de lente intraocular, manteniendo al paciente en posición decúbito ventral (8). Tornambe y colaboradores publicaron los resultados de un estudio piloto en ojos con agujero macular grado III o IV en los que se realizó facoemulsificación, cirugía de agujero macular más gas perfluoropropano $\left(\mathrm{C}_{3} \mathrm{~F}_{8}\right)$ al $15 \%$ evitando la posición supina durante10 días después de la cirugía (9). El 79\% de los casos tuvo éxito anatómico y el $48 \%$ logró una capacidad visual de 20/50.

El presente estudio evalúa los resultados obtenidos al realizar cirugía de agujero macular combinada con facoemulsificación y colocación de lente intraocular, además de intercambio fluido/gas repetido, sin mantener al paciente en posición boca abajo.

\section{SUJETOS, MATERIAL Y MÉTODOS}

Se realizó un estudio piloto, prospectivo y descriptivo en nuestro centro entre marzo de 2002 y junio de 2003.

Se incluyeron pacientes con diagnóstico de agujero macular a los cuales se les interrogó sobre el tiempo de evolución basado en la presencia de la sintomatología, considerada con fines estadísticos como mayor o menor de 6 meses (disminución de la agudeza visual, metamorfopsias, escotoma central).

Los criterios de inclusión fueron pacientes de cualquier edad y sexo con diagnóstico clínico, fluorangiográfico y por tomografía óptica de coherencia de agujero macular idiopático, grados III y IV de cualquier tiempo de evolución, con o sin opacidad de cristalino, ésta fue graduada según la clasifica- 
ción de Thompson (7). Los criterios de exclusión fueron patología de vítreo o retina, afaquia, uveítis, glaucoma, patología corneal, miopía alta, y cirugía vitreorretiniana previa.

En todos los casos se practicó examen oftalmológico completo que incluyó valoración de la agudeza visual utilizando la cartilla de visión del ETDRS, evaluación en la lámpara de hendidura y oftalmoscopia indirecta. Además a los pacientes se les solicitó una angiografía fluoresceínica de retina, fotografía a color del polo posterior y tomografía óptica de coherencia.

\section{Tecnica quirúrgica}

Se realizó facoemulsificación por córnea clara con una incisión de 3,2 mm, seguido de colocación de un lente de acrílico plegable MA60BM (AcrySof, Alcon, Fort Worth, TX) en saco capsular. Posteriormente se realizó, por un mismo cirujano de vítreo y retina, vitrectomía vía pars plana a tres vías con separación y remoción de la corteza vítrea posterior del nervio óptico y del área macular. Con un micropick vitreorretiniano se identificó y levantó la membrana limitante interna la cual se removió con una pinza de mácula. En caso de reintervención se usará azul de tripán para confirmar la presencia o no de la membrana limitante interna. Finalmente, se realizó un intercambio fluido/aire y colocación de gas perfluoropropano $\left(\mathrm{C}_{3} \mathrm{~F}_{8}\right)$ a una concentración levemente expansible del $17 \%$ como tamponamiento intraocular prolongado. Se revisó la retina periférica en los $360^{\circ}$ con oftalmoscopio indirecto e indentación escleral. Se suturaron las esclerotomías y en el postoperatorio inmediato se pidió al paciente que se mantuviera con la cabecera elevada 45 grados. Se empleó estadística descriptiva y estadística no paramétrica.

\section{Manejo postoperatorio}

Los pacientes fueron examinados al primer día y cada semana del primer mes, posteriormente al segundo, cuarto, sexto y duodécimo mes del postoperatorio. Revisiones adicionales fueron dadas según las condiciones clínicas de cada paciente. En cada control postoperatorio se valoró el estado de la córnea, presión intraocular, estado del lente intraocular, tamaño de la burbuja intraocular medida en porcentaje, estado del agujero macular y del resto de la retina y presencia de complicaciones si éstas existían. La agudeza visual mejor corregida se valoró a partir del segundo mes del postoperatorio y en los controles sucesivos.

Durante las dos primeras semanas de seguimiento se observó el nivel de la burbuja de gas mediante oftalmoscopía indirecta, si se encontraba cerca del agujero macular se procedió a realizar un nuevo intercambio fluido/gas, esta vez, en el consultorio. Para esto primero se instiló anestesia tópica con proparacaína y se trató con solución tópica de tobramicina, se inyectó anestesia retrobulbar con 3 ml de xylocaína al $2 \%$ y el paciente se colocó en posición prona con un blefarostato en el ojo en cuestión.

En forma estéril en una jeringa de $20 \mathrm{ml}$ se aspiró a través de un filtro Millipore (Storz Instruments) $3,5 \mathrm{ml}$ de gas perfluoropropano $\left(\mathrm{C}_{3} \mathrm{~F}_{8}\right)$ y $17 \mathrm{ml}$ de aire, quedando una concentración levemente expansible del $17 \%$. Con una aguja estéril calibre $27 \mathrm{G}$, a 3 milímetros del limbo y en el meridiano de las VI se procedió a realizar el intercambio fluido/gas en forma completa controlando inmediatamente después la presión intraocular con tonometría de aplanación. Nuevamente se le pidió al paciente que se evitara la posición supina.

La evaluación postoperatoria del agujero macular fue considerado como éxito anatómico cuando el halo de desprendimiento se aplicó o cuando se aplicó y además el agujero macular se cerró.

\section{RESULTADOS}

Se incluyeron 20 ojos de 20 pacientes, 14 de sexo femenino y 6 masculino, entre los 60 y 75 años de edad con una mediana de 68 años (tabla I). La capacidad visual preoperatoria estuvo en un rango de 0,05 a 0,13 con una mediana de 0,06 . La agudeza visual postoperatoria se encontró en un rango de 0,05 a 0,3 con una mediana de 0,10 (fig. 1 ). Al comparar la agudeza visual inicial y final se encontró un resultado estadísticamente significativo $(\mathrm{p}=0,001)$.

Fue necesario realizar intercambio fluido/gas repetido a los 7 días del postoperatorio sólo en el $25 \%(\mathrm{n}=5)$ de los casos, ya que el resto no presentó un nivel importante de líquido intraocular que pudiera afectar el cierre del agujero.

El resultado anatómico final del agujero macular fue exitoso después de una cirugía en el $80 \%$ ( $\mathrm{n}=$ 16) de los casos, y con cirugía adicional el éxito 
Tabla I. Características demográficas de los pacientes

\begin{tabular}{|c|c|c|c|c|c|c|c|c|c|}
\hline \multirow{2}{*}{$\begin{array}{c}\text { Caso } \\
1\end{array}$} & \multirow{2}{*}{$\begin{array}{c}\text { Sexo } \\
\mathrm{H}\end{array}$} & \multirow{2}{*}{$\begin{array}{c}\text { Edad } \\
68\end{array}$} & \multirow{2}{*}{$\begin{array}{c}\begin{array}{c}\text { Tiempo } \\
\text { de evolucion }\end{array} \\
2\end{array}$} & \multirow{2}{*}{$\begin{array}{c}\begin{array}{c}\text { Grado } \\
\text { agujero macular }\end{array} \\
\text { IV }\end{array}$} & \multicolumn{2}{|c|}{$\begin{array}{l}\text { AV PREOPERATORIA } \\
\text { Decimal/Logmar }\end{array}$} & \multicolumn{2}{|c|}{$\begin{array}{c}\text { AV POSTOPERATORIA } \\
\text { Decimal/Logmar }\end{array}$} & \multirow[t]{2}{*}{ Complicaciones } \\
\hline & & & & & 0,06 & 1,2 & 0,25 & 0,6 & \\
\hline 2 & $\mathrm{M}$ & 68 & 1 & IV & 0,13 & 0,9 & 0,32 & 0,5 & \\
\hline 3 & M & 64 & 1 & IV & 0,08 & 1,1 & 0,13 & 0,9 & \\
\hline 4 & $\mathrm{H}$ & 60 & 2 & IV & 0,06 & 1,2 & 0,06 & 1,2 & \\
\hline 5 & $\mathrm{M}$ & 65 & 2 & III & 0,05 & 1,3 & 0,05 & 1,3 & OCP \\
\hline 6 & M & 71 & 1 & III & 0,08 & 1,1 & 0,10 & 1 & $\uparrow \mathrm{PIO}$ \\
\hline 7 & M & 75 & 2 & IV & 0,05 & 1,3 & 0,05 & 1,3 & \\
\hline 8 & $\mathrm{H}$ & 66 & 2 & IV & 0,05 & 1,3 & 0,10 & 1 & \\
\hline 9 & M & 72 & 1 & IV & 0,06 & 1,2 & 0,06 & 1,2 & \\
\hline 10 & M & 68 & 1 & IV & 0,10 & 1 & 0,13 & 0,9 & \\
\hline 11 & M & 66 & 2 & III & 0,05 & 1,3 & 0,10 & 1 & \\
\hline 12 & M & 69 & 1 & IV & 0,13 & 0,9 & 0,13 & 0,9 & $\begin{array}{l}\text { Deprendimiento } \\
\text { Retina }\end{array}$ \\
\hline 13 & $\mathrm{H}$ & 63 & 2 & III & 0,05 & 1,3 & 0,13 & 0,9 & \\
\hline 14 & M & 69 & 2 & IV & 0,06 & 1,2 & 0,06 & 1,2 & \\
\hline 15 & M & 71 & 1 & IV & 0,10 & 1 & 0,20 & 0,7 & \\
\hline 16 & $\mathrm{M}$ & 60 & 1 & IV & 0,08 & 1,1 & 0,20 & 0,7 & \\
\hline 17 & $\mathrm{H}$ & 74 & 2 & IV & 0,05 & 1,3 & 0,10 & 1 & \\
\hline 18 & M & 67 & 1 & IV & 0,05 & 1,3 & 0,13 & 0,9 & \\
\hline 19 & M & 64 & 1 & IV & 0,06 & 1,2 & 0,06 & 1,2 & \\
\hline 20 & $\mathrm{H}$ & 74 & 2 & IV & 0,10 & 1 & 0,20 & 0,7 & $\uparrow \mathrm{PIO}$ \\
\hline
\end{tabular}

Sexo: H (hombre), M (mujer); Tiempo de evolucion 1: <6 meses, 2: >6 meses; OCP Opacidad de la cápsula posterior; $\uparrow$ PIO Aumento de la presión intraocular.

total fue del $90 \%(\mathrm{n}=18)$. De este $90 \%$ que presentaron cierre real del agujero, un $27 \%(\mathrm{n}=5)$ no presentaron variación en la agudeza visual. Al comparar el estado anatómico inicial del agujero macular con el final se obtuvo un resultado estadísticamente significativo con una $\mathrm{p}<0,001$ (tabla II).

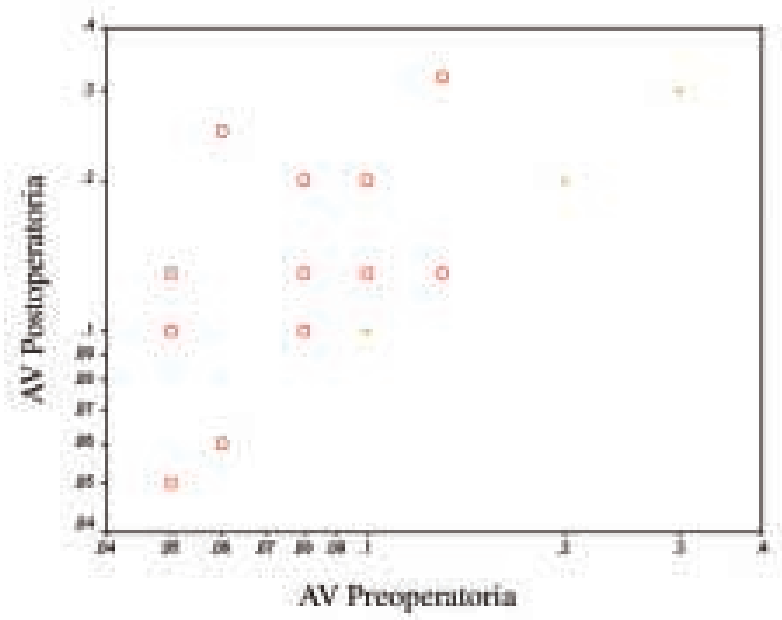

Fig. 1: Agudeza visual preoperatoria vs postoperatoria (Decimal). Los círculos rojos representan los casos, las cruces verdes marcan la línea de igual agudeza visual.
No se encontró asociación estadísticamente significativa al comparar el tiempo de evolución ni el grado del agujero macular con el resultado final de

Tabla II. Estado anatómico del agujero macular

\begin{tabular}{|c|c|c|c|}
\hline Paciente & $\begin{array}{c}\text { Grado del agujero } \\
\text { macular }\end{array}$ & Reintervenciones & $\begin{array}{l}\text { Estado final } \\
\text { del agujero }\end{array}$ \\
\hline 1 & IV & & cerrado \\
\hline 2 & IV & & cerrado \\
\hline 3 & IV & & cerrado \\
\hline 4 & IV & peeling + IFG & cerrado \\
\hline 5 & III & & cerrado \\
\hline 6 & III & IFG & cerrado \\
\hline 7 & IV & & cerrado \\
\hline 8 & IV & & cerrado \\
\hline 9 & IV & IFG, peeling + IFG & abierto (BR) \\
\hline 10 & IV & & cerrado \\
\hline 11 & III & peeling + IFG & abierto (BR) \\
\hline 12 & IV & & cerrado \\
\hline 13 & III & & cerrado \\
\hline 14 & IV & IFG & cerrado \\
\hline 15 & IV & & cerrado \\
\hline 16 & IV & IFG & cerrado \\
\hline 17 & IV & & cerrado \\
\hline 18 & IV & & cerrado \\
\hline 19 & IV & IFG, peeling + IFG & cerrado \\
\hline 20 & IV & & cerrado \\
\hline
\end{tabular}

IFG = Intercambio fluido-gas con $\mathrm{C}_{3} \mathrm{~F}_{8} 17 \%$; peeling = segunda cirugía con tinción de MLI con azul tripán y limitorrexis; BR: Borde reaplicado. 
la agudeza visual $p=1,00$ y $p=0,96$ respectivamente (prueba de Wilcoxon).

Al evaluar el estado del cristalino encontramos que el $30 \%$ de los casos era grado 0 (transparente), el $50 \%$ presentaba grado 0,75 (catarata mínima) y el $20 \%$ presentaba grado 2,00 (moderada).

Se presentaron complicaciones en 6 casos (30\%). La complicación que se presentó con mayor frecuencia (10\%) fue la elevación de la presión intraocular que fue manejada con éxito con beta bloqueadores. Se presentó un caso con desprendimiento de retina regmatógeno en el sector inferior, el que se manejó con exoplante. Un caso desarrolló opacificación mínima de la cápsula posterior que no requirió capsulotomía con láser YAG. Y en un caso hubo alteraciones en el epitelio pigmentario.

\section{DISCUSIÓN}

La vitrectomía con uso de tamponamiento con gas perfluoropropano $\left(\mathrm{C}_{3} \mathrm{~F}_{8}\right)$ se ha convertido en una forma aceptada para el tratamiento del agujero macular. El mecanismo por el cual un agujero macular se cierra después de vitrectomía no es claro, algunos estudios histopatológicos han demostrado un aparente acercamiento de los bordes del agujero y presencia de gliosis $(10,11)$.

Thompson en 1997 describió que los casos operados que presentan una evolución crónica (igual o mayor de dos años) tienen mayor dificultad en cerrar y peor pronóstico visual (12). Roth, en cambio presentó resultados anatómicos en los agujeros maculares crónicos similares a los de aparición reciente pero con mejores resultados funcionales en estos últimos (13). En nuestro estudio se encontró una mejoría de la agudeza visual estadísticamente significativa ( $\mathrm{p}=0,001)$, con un seguimiento mínimo de 12 meses, sin embargo, no se encontró asociación de la agudeza visual final con el tiempo de evolución o el grado de agujero macular. Aunque algunos autores describieron que los pacientes con cirugía de agujero macular continuaban ganando visión y disminución del escotoma central, después de un año de operado (6).

Aunque no se ha demostrado su utilidad, se ha generalizado la posición prona estricta en el postoperatorio de la cirugía de agujero macular por periodos que varían de dos a tres semanas, permitiendo que el gas perfluoropropano $\left(\mathrm{C}_{3} \mathrm{~F}_{8}\right)$ esté más tiempo en contacto con el agujero macular. Tam- bién se ha justificado esta posición para proteger al cristalino del efecto cataratogénico del gas. Sin embargo, el hecho de que la población afectada por esta patología sea en su gran mayoría adultos mayores de sesenta años de edad la hace extremadamente difícil de llevar a cabo por el tiempo requerido.

Como ya fue descrito nuestra técnica varió al prolongar el efecto de tamponamiento del gas, realizando un intercambio fluido/gas repetido a los 7 días del postoperatorio, o cuando el nivel del líquido intraocular se acercaba al agujero macular. Según nuestros resultados esto no fue necesario en todos los casos, y no consideramos al intercambio fluido/gas como tratamiento indicado en los casos fallidos, ya que en éstos preferimos realizar un nuevo pelado del agujero. Sin embargo, otros autores como Johnson y colaboradores en 1997 al realizar un intercambio fluido/gas temprano en 23 casos de cirugía de agujero macular fallido, dentro de una a ocho semanas de postoperatorio, obtuvieron cierre del agujero macular en un 74\% (14).

Ohana en 1998 publicó sus resultados para tratar agujero macular reabierto grado III o IV en 15 ojos previamente vitrectomizados. Se les realizó una fotocoagulación del epitelio pigmentado de la retina en los márgenes del agujero y un intercambio fluido/gas en el consultorio, con resultados del $80 \%$ de éxito anatómico con un sólo procedimiento (15).

Thompson y colaboradores en 1998 publicaron sus observaciones al usar factor de crecimiento tisular recombinante $\beta$ (TGF-beta2) y placebo en 130 ojos, sin hallar diferencia estadísticamente significativa entre los dos grupos tanto en el éxito anatómico como en el funcional (16). Consideramos injustificado el empleo de estas sustancias en nuestra técnica, sin embargo, existen múltiples artículos de empleo de concentrados plaquetarios en diferentes grados de agujero macular con buenos resultados $(17,18)$. Recientemente, Paques y colaboradores encontraron que los concentrados plaquetarios tienen un efecto importante en el cierre anatómico del agujero macular, no así en la agudeza visual final (19), por lo que no consideramos peligroso el hecho de dejar mínimas hemorragias maculares iatrogénicas transoperatorias. Se encontró aumento de la presión intraocular sólo en dos casos, que se resolvieron sin secuelas. Esta complicación es frecuente y de origen multifactorial, por ejemplo, por expansión de la burbuja de gas intraocular, inflamación del trabéculo, cierre angular o bloqueo pupilar, y ocurre generalmente dentro de la primera semana 
postoperatoria (20). Sin embargo, en la mayoría de los casos el aumento de la presión intraocular es transitorio, lo que también ocurrió en nuestra serie.

Se presentaron cambios en el epitelio pigmentado de la retina en un caso; se ha descrito que la incidencia es del 0 al 33\% dependiendo de la serie. Poliner y colaboradores en 1992, describieron un $75 \%$ de éxito anatómico $(\mathrm{n}=12)$, encontrando en todos ellos cambios en el epitelio pigmentado de la retina. Concluyeron que la combinación del contacto prolongado del gas y la exposición a la luz son los responsables de los cambios pigmentarios (21). La incidencia de desprendimiento de retina varía entre el 2 al 14\% siendo su causa los desgarros retinianos periféricos con tracción vítrea (22). Se recomienda realizar una vitrectomía lo más completa posible de tal manera que al momento de llenar el ojo con gas, éste no produzca tracción vítrea (23).

La cirugía de agujero macular es generalmente beneficiosa y los pacientes pueden mejorar su visión. La inyección de gas como tamponamiento y su asociación con la cirugía de catarata permite al paciente mantener una posición más confortable y tolerable sin que afecte el éxito de la cirugía. No está de más recordar que esta cirugía se asocia con complicaciones, por lo que se deben seleccionar cuidadosamente a los pacientes, y considerar que se trata de un procedimiento que aunque es reproducible y seguro, debe ser realizado por un cirujano con una amplia experiencia en cirugía de vítreo y retina.

\section{BIBLIOGRAFÍA}

1. Aaberg TM, Blair CJ, Gass JD. Macular holes. Am J Ophthalmol 1970; 69: 555-562.

2. Wendel RT, Patel AC, Kelly NE, Salzano TC, Wells JW, Novak GD. Vitreous surgery for macular holes. Ophthalmology 1993; 100: 1671-1676.

3. Gass JD. Idiopathic senile macular hole. Its early stages and pathogenesis. Arch Ophthalmol 1988; 106: 629-639.

4. Smiddy WE, Michels RG, Glaser BM, de Bustros S. Vitrectomy for impending idiopathic macular holes. Am J Ophthalmol 1988; 105: 371-376.

5. Kelly NE, Wendel RT. Vitreus surgery for idiopathic macular holes. Results of a pilot study. Arch Ophthalmol 1991; 109: 654-659.

6. Orellana J, Lieberman RM. Stage III macular hole surgery. Br J Ophthalmol 1993; 77: 555-558.

7. Thompson JT, Glasser BM, Sjaarda RN, Murphy RP. Progression of nuclear sclerosis and long-term visual results of vitrectomy with transforming growth factor beta-2 for macular holes. Am J Ophthalmol 1995; 119: 48-54.

8. MillerJH Jr, Googe JM Jr, Hoskins JC. Combined macular hole surgery and cataract surgery. Am J Ophthalmol 1997; 123: 705-707.

9. Tornambe PE, Poliner LS, Grote K. Macular hole surgery without face-down positioning. A pilot study. Retina 1997; 17: 179-185.

10. Funata M, Wendel RT, de la Cruz Z, Green WR. Clinicopathologic study of bilateral macular holes treated with pars plana vitrectomy and gas tamponade. Retina 1992; 12: $289-298$

11. Madreperla SA, Geiger GL, Funata M, de la Cruz Z, Green WR. Clinicopathologic correlation of a macular hole treated by cortical vitreous peeling and gas tamponade. Ophthalmology 1994; 101: 682-686.

12. Thompson JT, Sjaarda RN, Lansing MB. The results of vitreous surgery for chronic macular holes. Retina 1997; 17: 493-501.

13. Roth DB, Smiddy WE, Feuer W. Vitreous surgery for chronic macular holes. Ophthalmology 1997: 104: 2047-2052.

14. Johnson RN, Mc Donald HR, Schatz H, Ai E. Outpatient postoperative fluid-gas exchange after failed vitrectomy surgery for macular hole. Ophthalmology 1997; 104: 2009-2013.

15. Ohana E, Blumenkranz, MS. Treatment of reopened macular hole after vitrectomy by laser and outpatient fluid-gas exchange. Ophthalmology 1998; 105: 1398-1403.

16. Thompson JT, Smiddy WE, Williams GA, Sjaarda RN, Flynn HW Jr, Margherio RR, et al. Comparison of recombinant transforming growth factor-beta-2 and placebo as an adjunctive agent for macular hole surgery. Ophthalmology 1998; 105: 700-706.

17. Faude F, Edel E, Dannhauer M, Petzel C, Meier P, Wiedemann $P$. Autologous thrombocyte administration in treatment of idiopathic macular foramen. Ophthalmologe 1997; 94: 877-881.

18. Minihan M, Goggin M, Cleary PE. Surgical management of macular holes: results using gas tamponade alone, or in combination with autologous platelet concentrate, or transforming growth factor beta 2. Br J Ophthalmol 1997; 81: 1073-1079.

19. Paques M, Chastang C, Mathis A, Sahel J, Massin P, Dosquet $C$ et al. Effect of autologous platelet concentrate in surgery for idiopathic macular hole: results of a multicenter, double-masked, randomized trial. Platelets in Macular Hole Surgery Group. Ophthalmology 1999; 106: $932-$ 938.

20. Chen CJ. Glaucoma after macular hole surgery. Ophthalmology 1998; 105: 94-100.

21. Poliner LS, Tornambe PE. Retinal pigment epitheliopathy after macular hole surgery. Ophthalmology 1992; 99: 1671-1677.

22. Sjaarda RN, Glaser BM, Thompson JT, Murphy RP, Hanham A. Distribution of iatrogenic retinal breaks in macular hole surgery. Ophthalmology 1995; 102: 1387-1392.

23. Hutchins RK. Complications of macular hole surgery. Ophthalmology 1998; 105: 762. 